Article

\title{
An Innovative Metaheuristic Strategy for Solar Energy Management Through a Neural Framework
}

\author{
Hossein Moayedi 1,2*, Amir Mosavi ${ }^{3,4}$ * \\ ${ }^{1}$ Institute of Research and Development, Duy Tan University, Da Nang, 550000, Viet Nam, \\ hosseinmoayedi@duytan.edu.vn \\ 2 Faculty of Civil Engineering, Duy Tan University, Da Nang 550000, Vietnam \\ 3 School of Economics and Business, Norwegian University of Life Sciences, 1430 Ås, Norway; \\ a.mosavi@ieee.org \\ 4 John von Neumann Faculty of Informatics, Obuda University, 1034 Budapest, Hungary
}

Corresponding author: hosseinmoayedi@duytan.edu.vn; a.mosavi@ieee.org;

\begin{abstract}
Proper management of solar energy, as an effective renewable source, is of high importance toward sustainable energy harvesting. This paper offers a novel sophisticated method for predicting solar irradiance (SIr) from environmental conditions. To this end, an efficient metaheuristic technique, namely electromagnetic field optimization (EFO) is employed for optimizing a neural network. This algorithm quickly mines a publicly available dataset for nonlinearly tuning the network parameters. To suggest an optimal configuration, five influential parameters of the EFO (i.e., Npop, R_rate, Ps_rate, P_field, and N_field) are optimized by an extensive trial and error practice. Analyzing the results showed that the proposed model can learn the SIr pattern and predict it for unseen conditions with high accuracy. Furthermore, it provided about $10 \%$ and $16 \%$ higher accuracy compared to two benchmark optimizers, namely shuffled complex evolution and shuffled frog leaping algorithm. Hence, the EFO-supervised neural network can be a promising tool for the early prediction of SIr in practice. The findings of this research may shed light on the use of advanced intelligent models for efficient energy development.
\end{abstract}

Keywords: Power plant; Electrical power modeling; Metaheuristic strategy; Water cycle algorithm.

\section{Introduction}

Today, solar energy (SE) is considered a promising renewable source because of its advantages such as inexhaustible supply, environmental friendliness, universality, and high capacity [1, 2]. Indeed, the term artificial intelligence, is known as intelligence demonstrated by machines, (i.e., unlike the natural intelligence which involves emotionality and consciousness) shown by animals and humans. In this sense, the artificial intelligence models have provided a high competency for undertaking complicated and non-linear calculations [3-5]. Most recently, a number of artificial intelligence-based examples are studied such as in the subjects of environmental concerns [6-14], sustainability [15], pan evaporation and soil precipitation prediction [16-21], optimizing energy systems [22-30], natural gas consumption [31-33], water and groundwater supply chains [9, 34-43], image classification and processing, target tracking and computer vision [44-51], building and structural design analysis [52-56], quantifying climatic contributions [57], measurement techniques [44, 58-60], structural material (e.g., steel and concrete) behaviors [61-64], or even some complex concerns such as signal processing as well as feature selection and extraction problems [65-71]. There have been many decision-making applications works related to engineering complex problems as well $[54,72,73]$. A neural network is known as a series of complex algorithms that helps to recognize underlying connections in a set of data input and outputs through a process that mimics the way the 
human brain operates [74-78]. In another sense, the technique of artificial neural network (ANN) is a sophisticated nonlinear processor that has attracted massive attention for sensitive engineering modeling [79]. This model is represented by different notions. Most importantly, a multi-layer perceptron (MLP) $[80,81]$ is composed of a minimum of three layers, each of which contains some neurons for handling the computations-noting that a more complicated ANN-based solution is known as deep learning where it refers as part of a broader family of machine learning methods based on ANN with representation learning [51, 82-85]. For instance, Chen, et al. [86], Hu, et al. [87], Wang, et al. [88], and Xia, et al. [89] employed the use of extreme machine learning techniques on the field of medical sciences.

Having a reliable forecast of solar irradiance (SIr) is of great importance, due to its effect on the design of photovoltaic systems and measuring solar energy production [90, 91]. Figure 1 shows solar radiation on a photovoltaic module installed on the earth. Up to now, scholars have suggested various methods (e.g., empirical [92] and remote sensing [93] approaches) for analyzing the SE parameter. But recent advances in soft computing have led to the utilization of diverse machine learning tools for this purpose. These modes have gained a lot of attention for renewable energy analysis like feature selection [94].

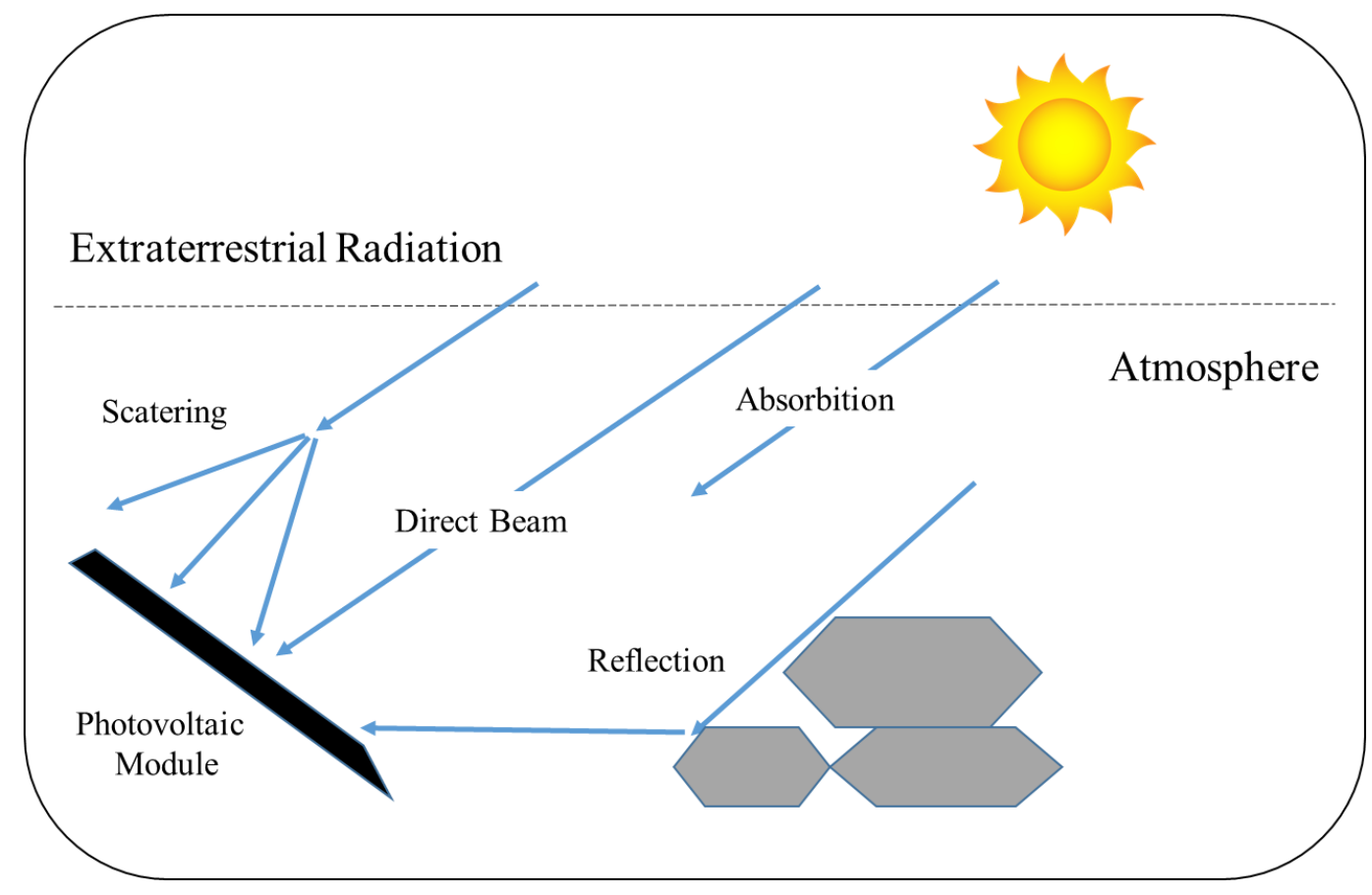

Figure 2. A schematic view of solar radiation and the SE production.

Artificial neural network (ANN), for example, is a flexible type of machine learning that has been broadly used for prediction tasks. Barrera, et al. [95] proposed an ANN model developed with open data sources for analyzing SE and also the effect of environmental factors on this parameter. The used model was found to be more accurate than previous methods (with MSE of 0.040 vs. 0.055). Yaïci, et al. [96] demonstrated the effectiveness of ANN for simulating the SE systems. They also investigated the effect of the problem dimension (i.e., the number of inputs) on the accuracy, and after testing the model using real-world (Ottawa, Canada) data, they professed that the accuracy falls gradually with reducing the dimension. Yadav, et al. [97] conducted a comparison among different ANN models, namely fitting tool (nftool), radial basis function neural network (RBFNN), and generalized regression neural network (GRNN) for analyzing the potential of SE resources in India. 
They reported the superiority of the nftool as it could nicely predict the desired parameter for many locations.

Meenal and Selvakumar [98] studied and demonstrated the accuracy of a popular machine learning called support vector machine (SVM) for solar radiation modeling. This method, when implemented with an optimal dataset, outperformed the ANN and empirical approaches for this purpose. Mohammadi, et al. [99] performed a feature analysis using another well-known processor, namely adaptive neuro-fuzzy inference system (ANFIS) for global solar radiation modeling. Quej, et al. [100] compared the potential of ANN, SVM, and ANFIS for simulating daily solar radiation. Concerning the respective average correlations of $0.652,0.689$, and 0.645 obtained for the best models, the SVM emerged as the most reliable predictor.

Metaheuristic algorithms have paved the way for more powerful forecasting models that are basically using the skeleton of conventional tools like ANN and ANFIS. These algorithms have been popularly used for renewable energy analysis [101] like wind energy [102], and more particularly, the SE-related simulations [103, 104]. In such methodologies (i.e., metaheuristic-based hybrids) optimal parameters are provided for the basic predictive method to avoid issues like local minima [105].

Abedinia, et al. [106] designed a forecast engine based on a metaheuristic optimizer called shark smell optimization combined with ANN for approximating solar power. Due to the better performance of this model in comparison with conventional predictors like conventional ANN, RBFNN, GRNN, and their wavelet versions (normalized root mean square errors (RMSEs) around 11 vs. those above 14), they introduced it as a capable engine. Galván, et al. [107] benefitted from a multiobjective particle swarm optimization (PSO) technique for optimizing the intervals of the SE modeling. They built a non-linear method using ANN and their findings revealed the high applicability of the PSO optimizer for the mentioned objective. Likewise, Halabi, et al. [108] could effectively use this algorithm coupled with an ANFIS system for monthly solar radiation approximation. Vaisakh and Jayabarathi [109] suggested a hybrid of two methods, namely deer hunting optimization algorithm and grey wolf optimization for tuning the structure of various ANNs applied to SIr forecast. Their results showed a promising improvement attained by the proposed optimizer. Louzazni, et al. [110] showed the competency of firefly algorithm for analyzing the parameters of the photovoltaic system under different conditions. Compared to previously-used metaheuristic techniques, the firefly algorithm achieved reliable and valid results in tuning the photovoltaic parameters. The efficiency of the PSO and genetic algorithm for a similar objective was demonstrated by Bechouat, et al. [111]. Wind driven optimization was successfully used by Abdalla, et al. [112] to dealing with the optimal power tracking of photovoltaics systems. This algorithm performed more efficiently than several optimization techniques such as the PSO, bat algorithm, cuckoo search, etc.

According to the explained literature, metaheuristic algorithms can yield promising solutions to complex issues like SIr prediction. But a gap of knowledge emerges when earlier studies have mostly used well-established strategies like PSO [113], GA [114], and imperialist competitive algorithm [115]. Furthermore, these techniques take a noticeable time to reach stable optimization. This study, therefore, focuses on a novel metaheuristic strategy, namely electromagnetic field optimization (EFO) for the optimal prediction of the SIr. A significant advantage of this algorithm is its fast convergence relative to other existing techniques. The EFO supervises a non-linear problem through an ANN framework. Moreover, two other quick algorithms of shuffled complex evolution (SCE) and shuffled 
frog leaping algorithm (SFLA) are considered as benchmark methods to comparatively validate the efficiency of the EFO.

\section{Materials and Methods}

\subsection{Data provision}

For predicting the SIr, a publicly available dataset (provided by NASA and available on https://www.kaggle.com/dronio/SolarEnergy) is used in this work. Prior to this study, this data has been used for validating the performance of different developed models [116, 117]. The SIr plays the role of the target parameter to be predicted with the inputs of temperature $(\mathrm{T})$, barometric pressure $(\mathrm{BP})$, humidity $(\mathrm{H})$, wind direction (WD), and wind speed (WS).

The used dataset contains 32686 rows of meteorological records obtained from the HI-SEAS weather station. With around 5 minutes intervals, the records belong to the time between 23:55:26 29 September 2016 to 00:00:02 1 December 2016. Figure 2 shows the variation of the SIr over one day (29 September 2016 taken as an instance). As expected, peak values are observed the midday. Moreover, Figure 3 depicts the relationship between the SIr and each input factor in the form of scatter charts for the whole data.

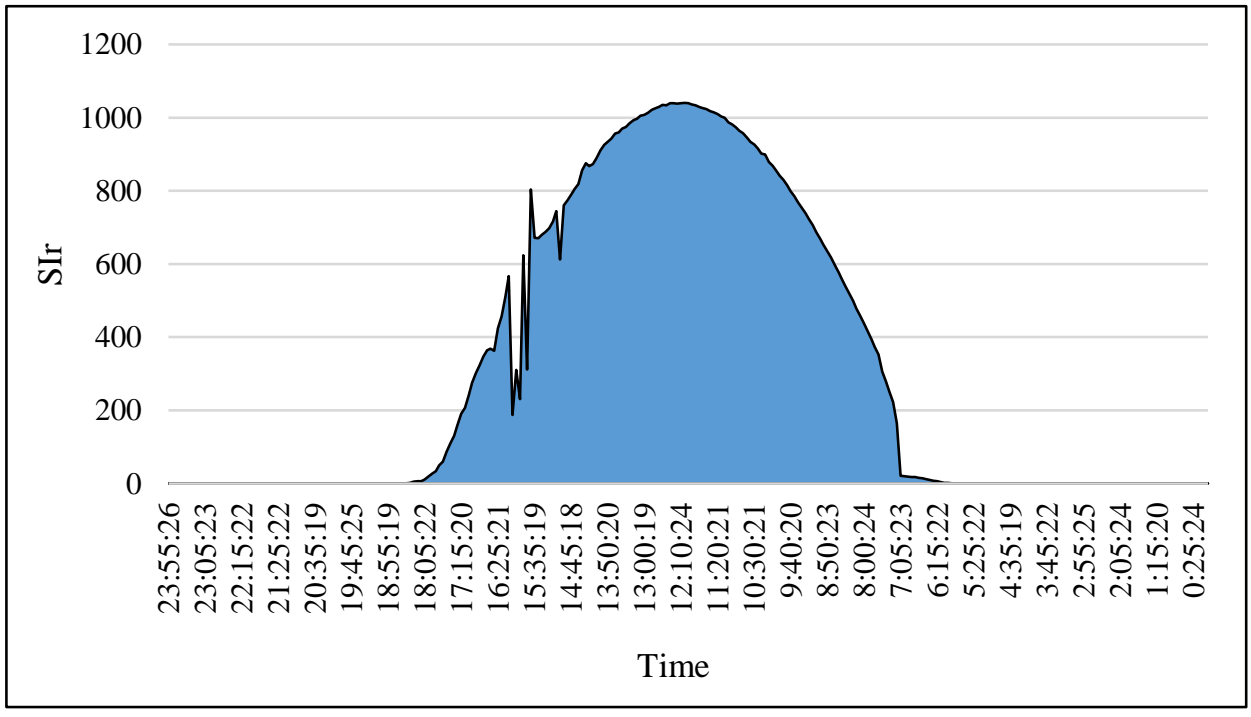

Figure 2. The variation of the SIr over 29 September 2016.

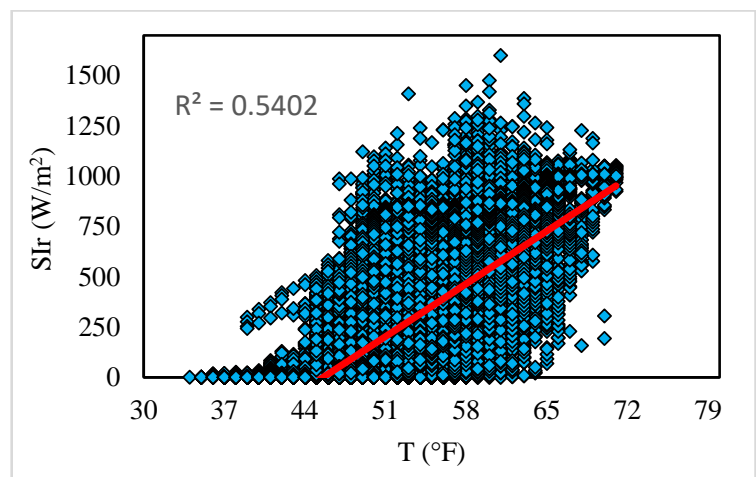

(a)

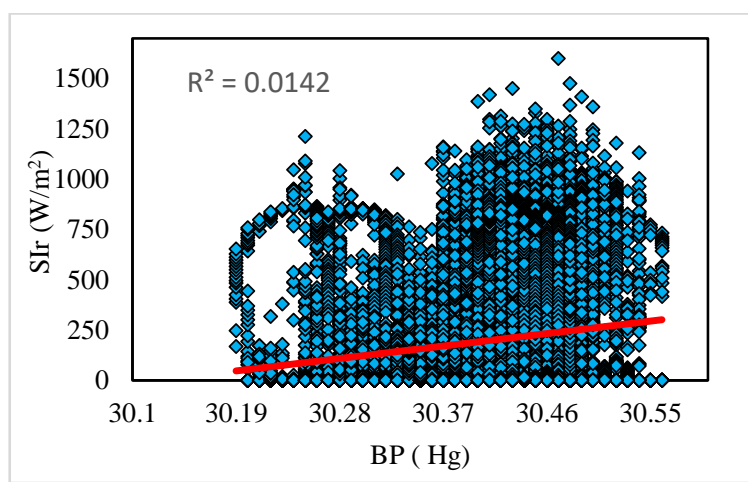

(b) 


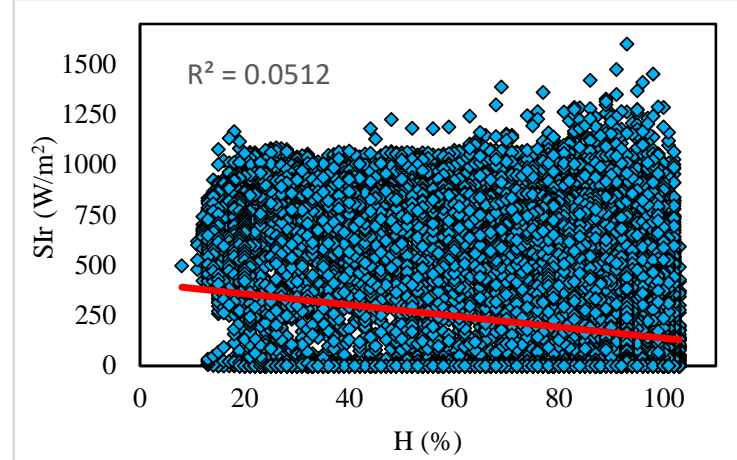

(c)

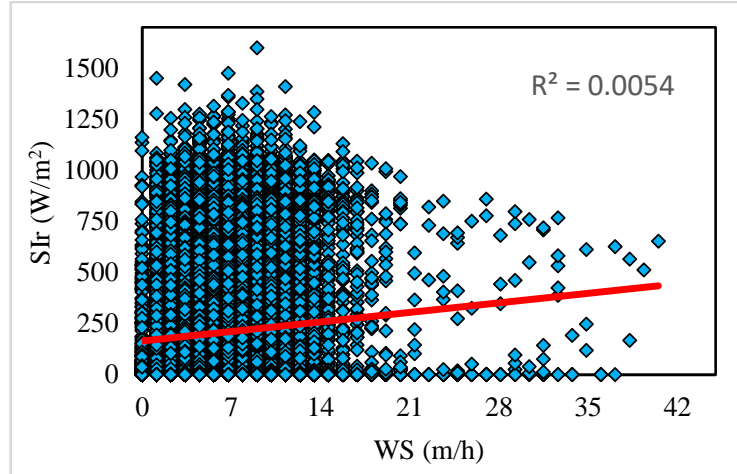

(e)

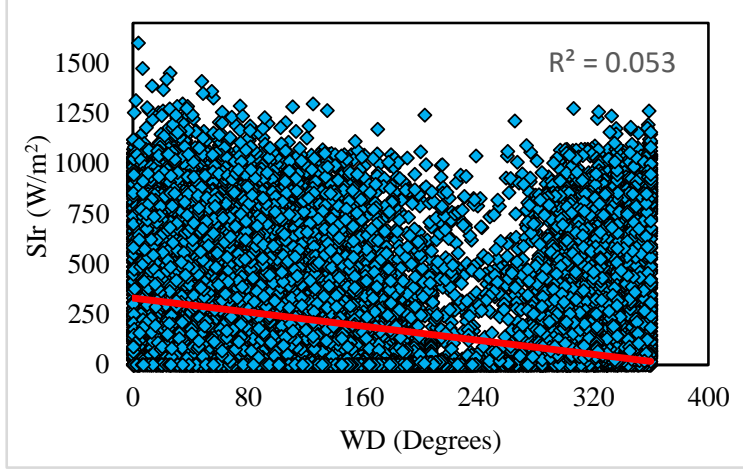

(d)

Figure 3. Scatter plots of the SIr versus input parameters.

Considering R2 values calculated in Figure 3 (0.5402, 0.0142, 0.0512, 0.053, and 0.0054 for the T, $\mathrm{BP}, \mathrm{H}, \mathrm{WD}$, and WS, respectively), it can be said that the most meaningful relationship (among these five inputs) is obtained for the T. In a general view, the values of SIr tend to increase with the increase in this factor. A detailed statistical description of the used dataset is presented in Table 1. As is seen, SIr values ranges in $[1.1,1601.3] \mathrm{W} / \mathrm{m} 2$, while this extend is $\left[34.0,71.0{ }^{\circ} \mathrm{F},[30.2,30.6] \mathrm{Hg}\right.$, [8.0, 103.0] $\%,[0.1,360.0]$ degree, and $[0.0,40.5] \mathrm{m} / \mathrm{h}$ for the T, BP, H, WD, and WS, respectively.

Table 1. Descriptive statistics of the SIr and input parameters.

\begin{tabular}{cccccccc}
\hline & & \multicolumn{6}{c}{ Descriptive indicator } \\
\cline { 3 - 7 } Factor & Unit & Mean & $\begin{array}{c}\text { Std. } \\
\text { Error }\end{array}$ & $\begin{array}{c}\text { Std. } \\
\text { Deviation }\end{array}$ & $\begin{array}{c}\text { Sample } \\
\text { Variance }\end{array}$ & Minimum & Maximum \\
\hline T & ${ }^{\circ} \mathrm{F}$ & 51.1 & 0.0 & 6.2 & 38.5 & 34.0 & 71.0 \\
BP & $\mathrm{Hg}$ & 30.4 & 0.0 & 0.1 & 0.0 & 30.2 & 30.6 \\
H & $\%$ & 75.0 & 0.1 & 26.0 & 675.5 & 8.0 & 103.0 \\
WD & Degree & 143.5 & 0.5 & 83.2 & 6916.8 & 0.1 & 360.0 \\
WS & $\mathrm{m} / \mathrm{h}$ & 6.2 & 0.0 & 3.5 & 12.2 & 0.0 & 40.5 \\
SIr & $\mathrm{W} / \mathrm{m} 2$ & 207.1 & 1.7 & 315.9 & 99803.2 & 1.1 & 1601.3 \\
\hline
\end{tabular}

In artificial intelligence implementation, it is well-established that machines use a part (the majority) of instances for learning the existing input-target pattern. They then apply this pattern to the remaining instances for evaluating the prediction ability. For this study, the dataset (i.e., 32686 instances) is randomly divided into two groups with 26149 and 6537 instances $(80 \%$ and $20 \%$ of the whole) to generate the training and testing dataset, respectively.

\subsection{Methodology}




\subsubsection{The EFO}

Abedinpourshotorban, et al. [118] developed a physics-based optimization strategy and named it electromagnetic field optimization. Many scholars have benefited from this method for a wide range of problems $[119,120]$. It is a population-based technique in which each individual is represented by an electromagnetic particle (EMP). The EMPs are distinguished by different polarities. The attraction-repulsion rule is used to improve the solution by changing the position of the EMPs.

The steps of the EFO can be explained as follows:

Step 1: A set of EMPs are randomly generated and the fitness of each one is calculated. The particles are then sorted based on these fitnesses. Each particle is made of N_var electromagnets (tantamount to the number of the problem variables).

Step 2: This is dedicating to dividing the EMP population into three filed groups with negative, positive, and neutral polarities. The positive field group comprises the best-fitted individuals tunable by a so-called parameter " $\mathrm{P}_{-}$field", the negative field group comprises the worst-fitted individuals tunable by a so-called parameter "N_field", and the rest lie in the third group.

Step 3: Each repetition of the algorithm generates a new EMP. Once this EMP is better-fitted than the weakest one, it is considered as a part of the population and confiscates the position of weakest EMP. Figure 4 shows the generation process and determining the polarity of the new member.

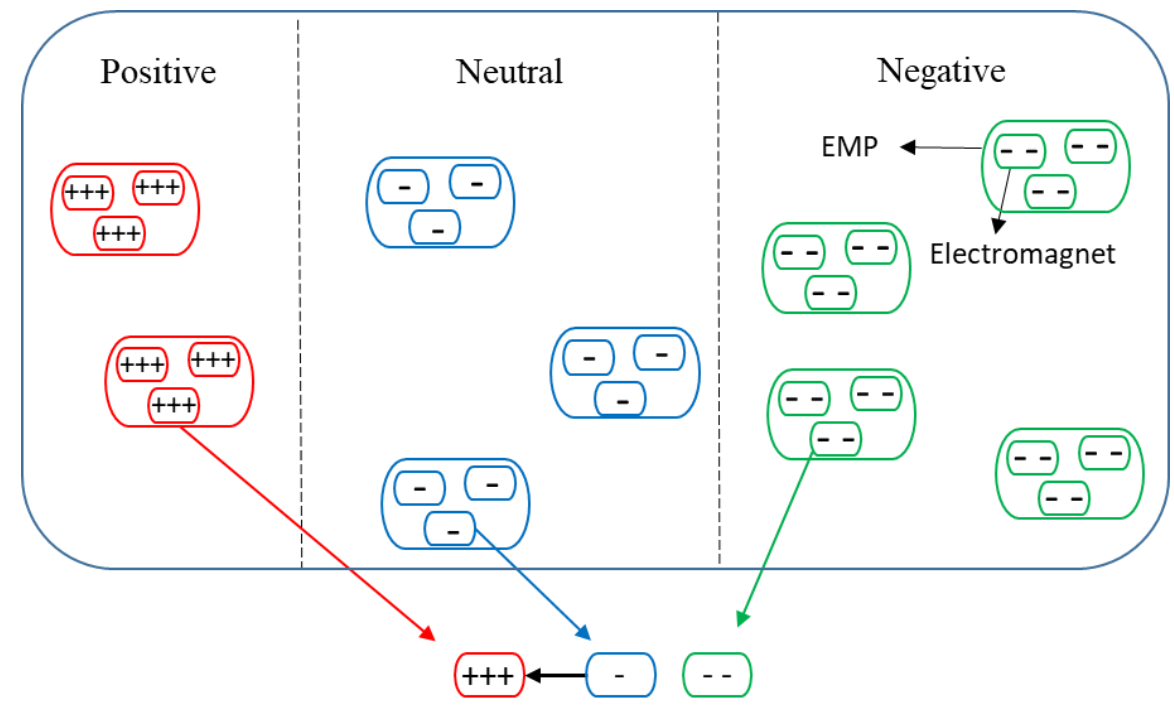

Figure 4. The creation of a new EMP.

In this process, for $\mathrm{j}=1 \rightarrow \mathrm{N}_{-}$var, an electromagnet belonging to the neutral field group is chosen. Next, a random value is considered and compared to a parameter called Ps_rate which indicates the probability of choosing electromagnets of the created EMP from the positive field. Equation 1 is used for the situation random value $<$ Ps_rate, otherwise, Equation 2 expresses the generation process.

$$
\begin{gathered}
E M P_{j}^{\text {new }}=E M P_{j}^{P F_{j}}, \\
E M P_{j}^{\text {new }}=E M P_{j}+(G R * \text { rand }) \times\left(E M P_{j}^{P F_{j}}-E M P_{j}\right)+(\text { rand }) \times\left(E M P_{j}^{N F_{j}}-E M P_{j}\right),
\end{gathered}
$$

where PF and NF symbolize positive and negative fields, GR gives the golden ratio, rand is the random value inside [0, 1],

Step 4: A randomization operator is responsible for diversifying the new EMPs. Another random value is generated and compared to a parameter called $R \_$rate which indicates the probability of 
replacing one electromagnet of the created EMP with a random electromagnet. If random value $<$ R_rate, a new electromagnet replaces one electromagnet of the created EMP [120].

\subsubsection{The benchmarks}

The SCE and SFLA are efficient metaheuristic techniques that are used as comparative methods in this work. While both algorithms are based on shuffle action, the SCE is an older optimizer. Duan, et al. [121] and Eusuff and Lansey [122] presented the SCE and SFLA in 1993 and 2003, respectively. Although this study is one of the first usages of the EOF for supervising an ANN, scholars like Zheng, et al. [123] and Ma, et al. [124] have reported successful performance of the SCE and EFO for this purpose.

The SCE implements a combination of the Nelder-Mead simplex technique, genetic algorithm, complex shuffling, and controlled random search for doing the optimization. After creating the population, the individuals are grouped in some containers called complexes. The algorithm uses competitive complex evolution for evolving these complexes. It then synthesizes evolved units to create a larger community. This step results in more interactive agents for better sharing the obtained knowledge [125]. The pivotal idea of the SFLA is the relationship between frogs settled in some containers called memeplexes. It is known as a quick and efficient search scheme that synthesizes the PSO with the memetic algorithm. The fitness of the frogs is a measure for classifying them the memeplexes. The SFLA pursues updating the position of the frogs in these units, and also, importing new ones instead of the worst individuals [126]. The benchmark algorithms are mathematically detailed in earlier studies like $[127,128]$ (for the SCE) and $[129,130]$ (for the SFLA).

Similar to the EFO, two separate ANNs are supervised by the benchmark algorithms to explore and predict the SIr. The performance of these three methods is compared in the following sections to return an optimal metaheuristic-based methodology for this purpose.

\section{Results and discussion}

\subsection{Accuracy assessment measures}

The accuracy of SIr prediction is reported by well-known indices as follows: Given Error $_{i}=$ $S I r_{i_{\text {recorded }}}-S I r_{i_{\text {predicted }}}$, the error of prediction for a total of N instances is calculated by the RMSE and mean absolute error (MAE) indices. According to Equations 3 and 4, RMSE gives a rooted value of the averaged squared Errors, while the MAE releases an average of the absolute Error values.

$$
\begin{gathered}
\text { RMSE }=\sqrt{\frac{1}{N} \sum_{i=1}^{N}\left[\text { Error }_{i}{ }^{2}\right.}, \\
M A E=\frac{1}{N} \sum_{i=1}^{N} \mid \text { Error }_{i} \mid,
\end{gathered}
$$

Besides, a correlation index called Pearson correlation coefficient $(R)$ is also defined to show the consistency between the recorded SIrs and the products of each network. Equation 5 formulates the R: 


$$
R=\frac{\sum_{i=1}^{N}\left(S I r_{i_{\text {predicted }}}-\overline{S I r}_{\text {predicted }}\right)\left(S I r_{i_{\text {recorted }}}-\overline{S I r}_{\text {recorded }}\right)}{\sqrt{\sum_{i=1}^{N}\left(S I r_{i_{\text {predicted }}}-\overline{S I r}_{\text {predicted }}\right)^{2}} \sqrt{\sum_{i=1}^{N}\left(S I r_{i_{\text {recorled }}}-\overline{S I r}_{\text {recorded }}\right)^{2}}},
$$

where $\overline{\operatorname{SIr}}$ symbolizes the average of the SIr values.

\subsection{Optimization and training}

A $5 \times 45 \times 1$ MLP neural network (indicating 5 nodes in the input layer, 45 nodes in the middle layer, and 1 node in the output layer) is used to connect the SIr to its input factors. Due to a large number of data, this network is a complex system that is supposed to be supervised by the EFO algorithm. The main role of the EFO is to adjust the MLP internal parameters so that the SIr pattern is optimally established.

After creating the EFO-MLP hybrid, it is trained by mining the training group. Since metaheuristic algorithms are population-based iterative techniques, optimum values should be considered for these two parameters i.e., population size (Npop) and the number of iterations (NIt). Despite many optimization algorithms that reach a stable situation by around 1000 iterations, the EFO needs more effort. Based on experience and also evaluating the behavior of the model, the EFOMLP is implemented by a total of 50000 iterations. The appropriate values for Npop, as well as four other parameters, are determined one by one by testing different values. The convergence curves of the tested EFO-MLPs are shown in Figure 5. First the model with different Npops $(25,26,27,28,30,35$, and 40) are tested (when $R \_$rate $=0.01$, Ps_rate $=0.01, P \_$field $=0.02$, and N_field $=0.4$ ). Figure $5-(\mathrm{a})$ shows that the $\mathrm{N}_{\text {Pop }}=26$ gives the lowest error. Thus, the subsequent models are tested with this $\mathrm{N}_{\text {Pop. }}$. Five R_rates of $0.01,0.015,0.02,0.03$, and 0.04 are similarly assessed. According to Figure 5 - (b), $\mathrm{R} \_$rate $=0.01$ is the most suitable one. Next, investigating the effect of Ps_rate in Figure $5-(\mathrm{c})$ revealed that the lowest error is obtained for Ps_rate $=0.03$. As is exhibited in Figure $5-(\mathrm{d})$, $\mathrm{P} \_$field experienced the values of $0.02,0.03,0.04,0.05$, and 0.06 and $P_{-}$field $=0.02$ remained as the best value. Lastly, the values considered for N_field, 0.1, 0.2, 0.3, 0.4, and 0.5, are depicted in Figure 5 - (e) which demonstrates the lowest error for $N_{-}$field $=0.4$.

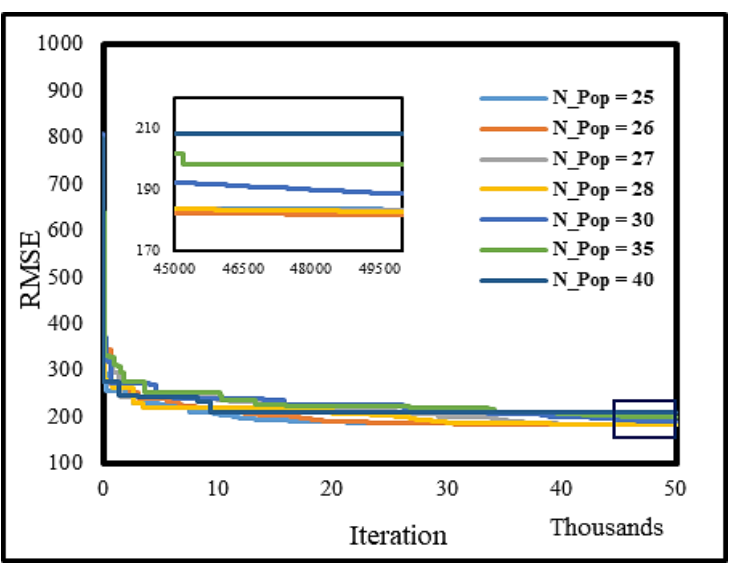

(a)

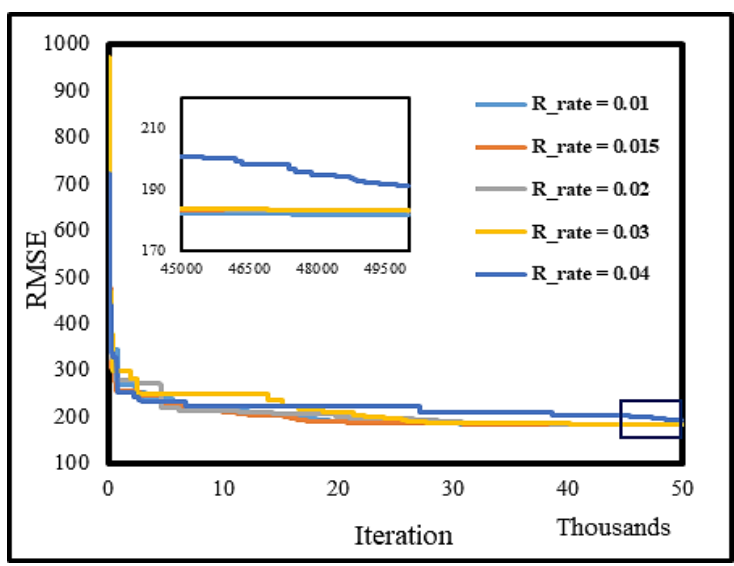

(b) 


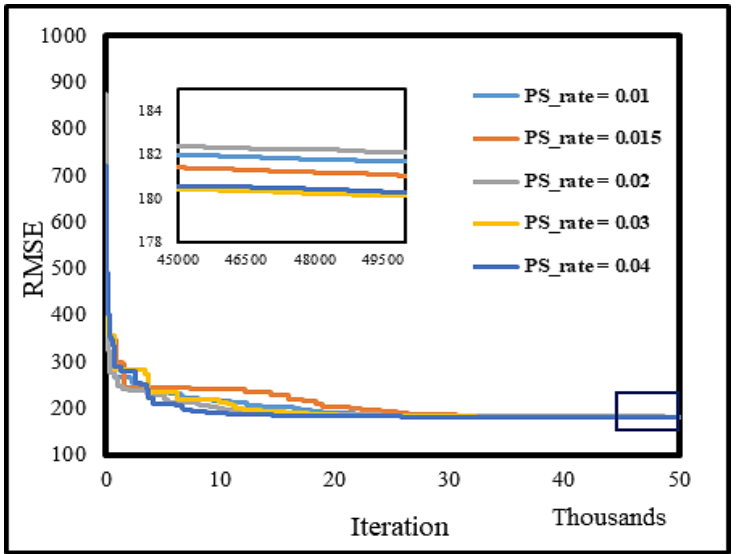

(c)

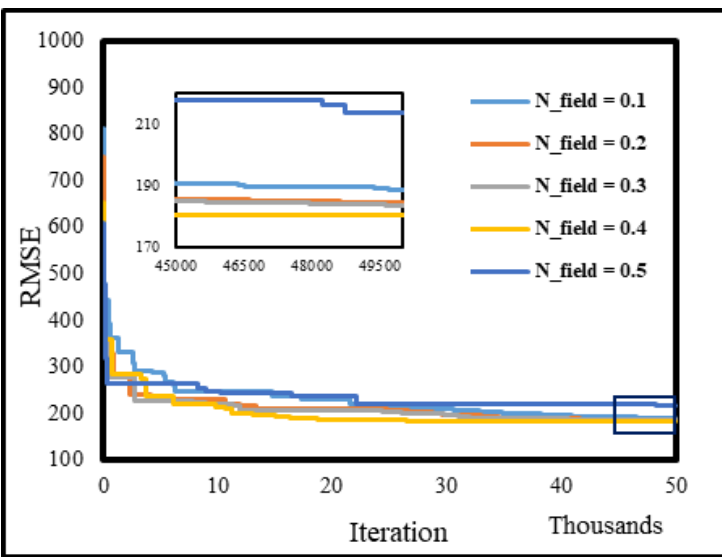

(e)

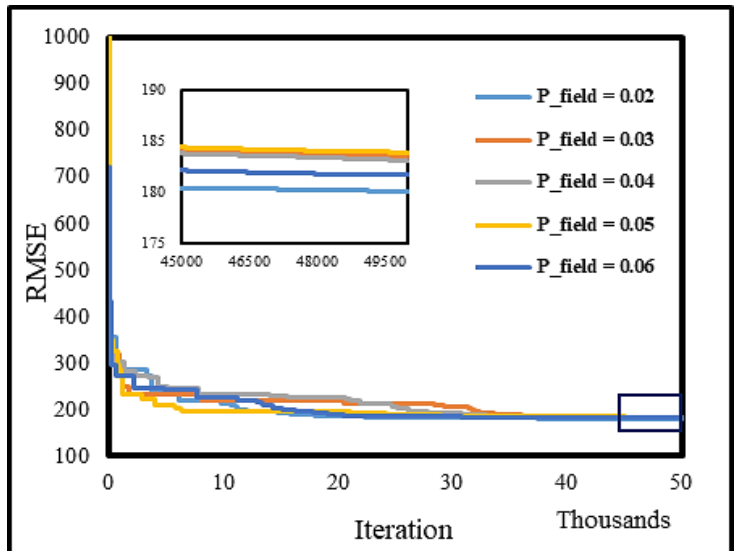

(d)

Figure 5. Optimizing the effect of EFO parameters including (a) Npop, (b) R_rate, (c) Ps_rate, (d) P_field, and (e) N_field.

A similar strategy was executed for the benchmark models (i.e., SCE-MLP and SFLA-MLP). Table 2 denotes the values assigned to the used algorithms. As is seen, the SCE and SFLA are implemented with 1000 iterations.

Table 2. Implementation parameters of the used algorithms.

\begin{tabular}{ccc}
\hline EFO & SCE & SFLA \\
\hline NPop $=26$ & NPop $=10$ & NPop $=25$ \\
R_rate $=0.01$ & No. of offsprings $=3$ & Step size $=1$ \\
Ps_rate $=0.01$ & No. of complexes $=3$ & No. of offsprings $=3$ \\
P_field $=0.02$ & NIt $=1000$ & No. of memeplexes $=5$ \\
N_field $=0.4$ & & NIt $=1000$ \\
NIt $=50000$ & & \\
\hline
\end{tabular}

Assessing the RMSEs obtained for the EFO-MLP, SCE-MLP, and SFLA-MLP, that are 180.1228, 197.4813, and 208.1472, respectively, shows that the used hybrids could learn the SIr pattern with good accuracy. The corresponding MAEs are 117.8681, 138.5814, and 156.2768 which, regarding the range of the observed SIrs (Table 1), indicate an acceptable level of error. Moreover, the correlation values of $0.82275,0.78208$, and 0.75431 demonstrate a high agreement between the training products and expected SIrs. 


\subsection{Testing results}

As explained in section 2, the second part of the dataset plays the role of unseen environmental conditions. The models use this data to evaluate their testing ability. In this regard, the SIr is forecasted for the testing instances and these values are compared with the expected values. Since the model does not perform any analysis on these instances, it has to use the previously captured knowledge. Accordingly, the goodness of the results reflects the prediction capability of the intended model.

Considering Error $_{i}$ formula (section 4.1), Figure 6 details the magnitude and statistics of Error values calculated for the testing instances. In this phase, the RMSEs of 177.9764, 195.0984, and 205.6091 indicated a reliable prediction by all three models. Moreover, the goodness of the testing results can be supported by the MAEs of $115.2678,136.2261$, and 154.1603, as well as the R values of $0.82132,0.78046$, and 0.75212 .

Moreover, from a graphical point of view, the histogram charts in Figure 6 show that the small Errors outnumber large values. It can be derived from the sharp shape of the diagram around zero and the vicinity. Regarding the overall trend of these charts, the magnitude of the Error increases as the frequency falls.

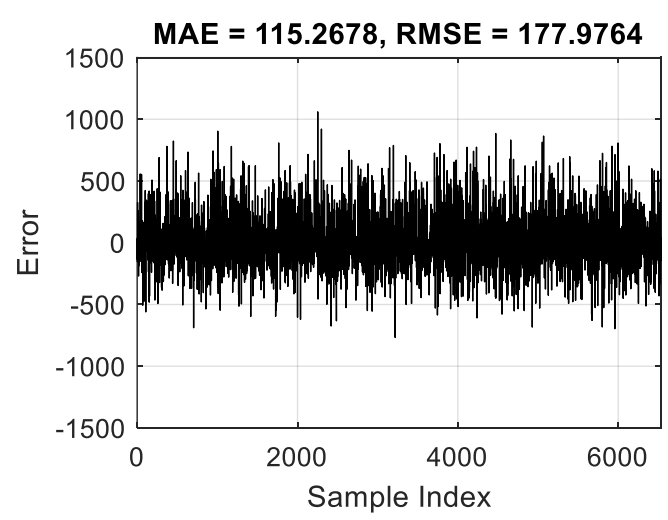

(a)

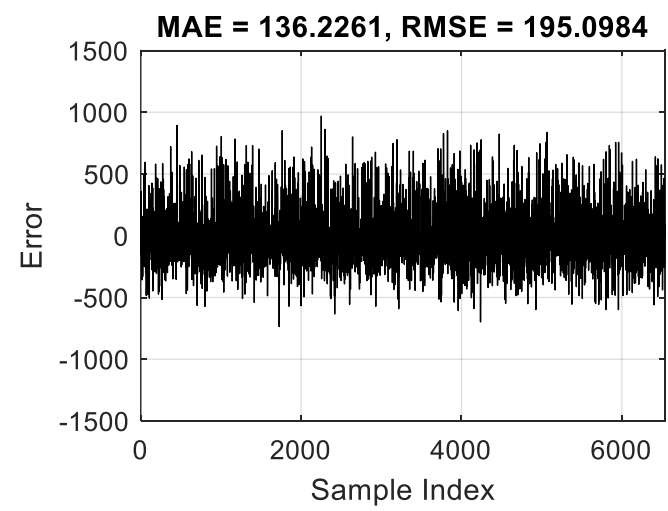

(c)

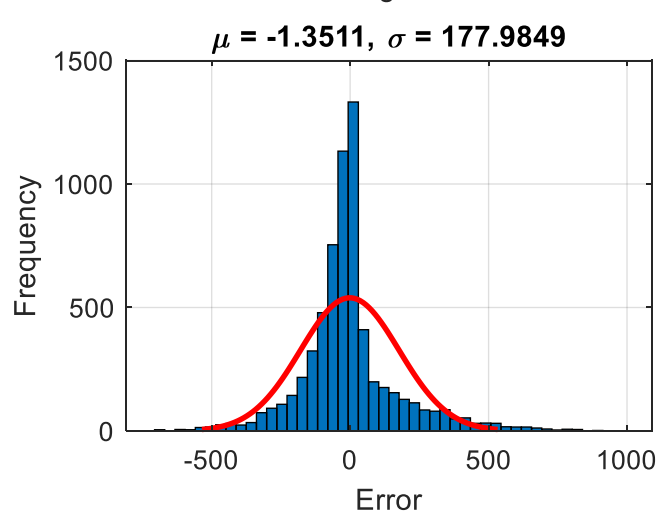

(b)

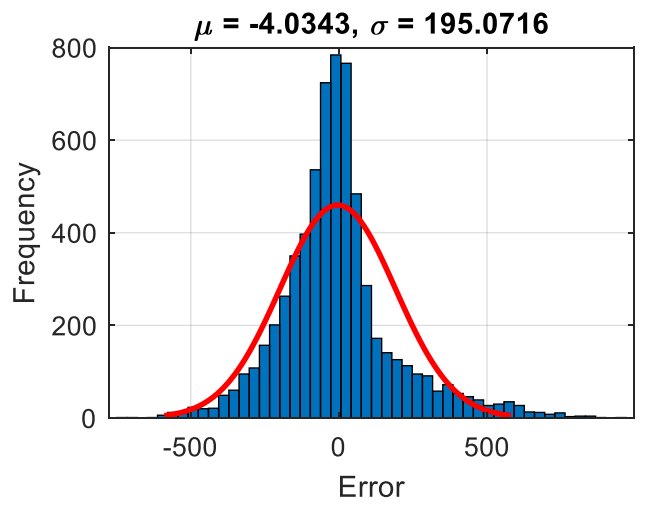

(d) 


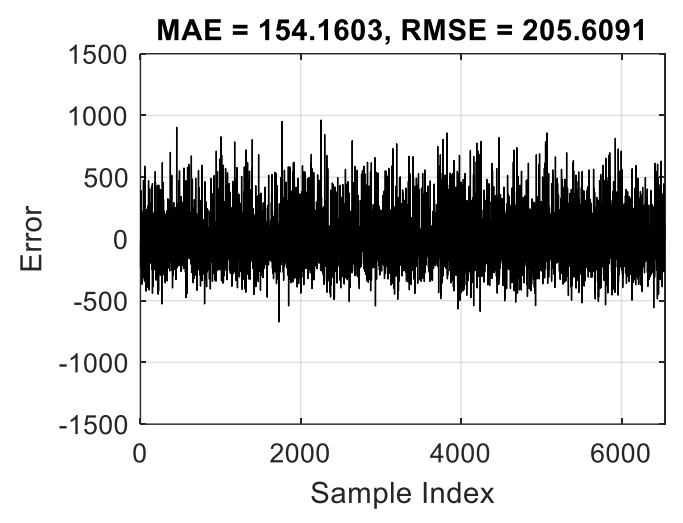

(e)

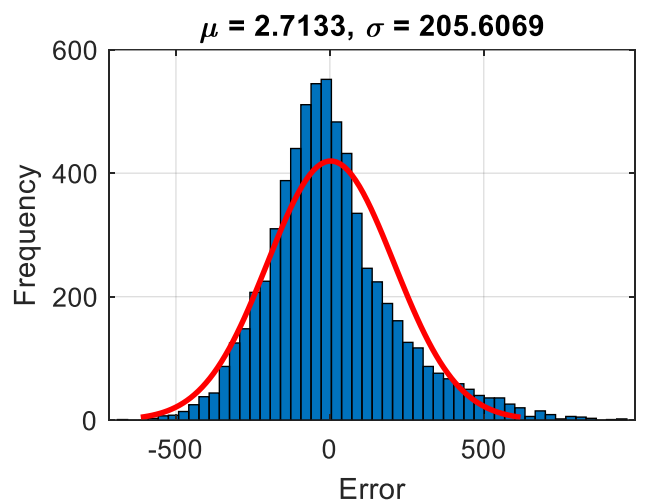

(f)

Figure 6. The Error analysis in terms of (a, c, and e) magnitude and (b, d, and f) frequency for the EFO-MLP, SCE-MLP, and SFLA-MLP, respectively.

\subsection{EFO vs. SCE and SFLA}

It was stated that this research pursues a novel time-efficient methodology for analyzing the SIr. The EFO was presented as the pivotal method, while the SCE and SFLA acted as benchmark algorithms. Earlier sections showed the competency of all three supervised models. Hence, this section validates the performance of the EFO versus the SCE and SFLA.

For both training and testing groups, the error indicators showed a lower error of prediction, and at the same time, the $\mathrm{R}$ index manifested a higher correlation for the EFO-trained model. Table 3 gives the accuracy improvements when the SCE and SFLA are replaced with the EFO. As is seen, in the case of EFO vs. SCE, the RMSE and MAE fall by nearly 10 and 18\% in both phases, respectively. Also, a 4\% enhancement resulted for the R index. As for EFO vs. SFLA, the changes are more tangible. The RMSE and MAE of both phases degrade by around 16 and 33\%, respectively. The R index indicated a $7 \%$ better correlation, too.

Table 2. Improvements achieved by the EFO algorithm vs. the benchmarks.

\begin{tabular}{ccccccc}
\hline & \multicolumn{3}{c}{ Improvements } \\
\cline { 2 - 7 } $\begin{array}{c}\text { Comparative } \\
\text { hybrid }\end{array}$ & \multicolumn{3}{c}{ Training phase } & \multicolumn{3}{c}{ Testing phase } \\
\cline { 2 - 7 } & RMSE (\%) & MAE (\%) & R & RMSE (\%) & MAE (\%) & R \\
\hline Vs. SCE & 9.64 & 17.57 & 0.04 & 9.62 & 18.18 & 0.04 \\
\hline Vs. SFLA & 15.56 & 32.59 & 0.07 & 15.53 & 33.74 & 0.07 \\
\hline
\end{tabular}

\section{Conclusions}

This research was dedicated to finding a fast yet reliable solution for predicting solar irradiance. Since this parameter is affected by different factors, the problem is a non-linear complex one. Therefore, a potent metaheuristic strategy called electromagnetic field optimization was considered for dealing with it. A neural network organized the general equations, while the EFO tuned their parameters optimally. Moreover, this algorithm was compared with two shuffle-based metaheuristic techniques, namely shuffled frog leaping algorithm and shuffled complex evolution. While a good 
level of accuracy was observed for all three hybrids, the EFO-MLP was significantly superior. For example, its error was around $10 \%$ and $16 \%$ below that of the SCE-MLP and SFLA-MLP, respectively. Referring to the $\mathrm{R}$ value of 0.82132 for testing data, the proposed model can reliably predict the SIr for given environmental conditions. Apart from the high implementation speed, another advantage of the used EFO-MLP model lies in implementation with optimized parameters (i.e., NPop, R_rate, Ps_rate, P_field, and N_field). Therefore, the findings of this study can be used for sustainable energy management. However, there may be still ideas for future works (e.g., using feature selection and filtrated data) for a more efficient methodology.

Author Contributions: Conceptualization, X.S. and N.Y.; methodology, X.S.; software, X.S.; validation, X.S.; writing - original draft preparation, X.S.; writing-review and editing, N.Y.; visualization, X.S. and N.Y.; supervision, N.Y.; project administration, N.Y. All authors have read and agreed to the published version of the manuscript.

Funding: This research was funded by the major project of philosophy and Social Sciences Application Research of Henan University "Research on evolution mechanism and credit evaluation of credit risk of start-up enterprises", grant number 2016-yyzd-11.

Conflicts of Interest: The authors declare no conflict of interest.

\section{References}

1. Gong, J.; Li, C.; Wasielewski, M. R., Advances in solar energy conversion. Chemical Society Reviews 2019, 48, (7), 1862-1864.

2. Mofijur, M.; Mahlia, T. M. I.; Silitonga, A. S.; Ong, H. C.; Silakhori, M.; Hasan, M. H.; Putra, N.; Rahman, S., Phase change materials (PCM) for solar energy usages and storage: an overview. Energies 2019, 12, (16), 3167.

3. Liu, Z.; Shao, J.; Xu, W.; Chen, H.; Zhang, Y., An extreme learning machine approach for slope stability evaluation and prediction. Natural hazards 2014, 73, (2), 787-804.

4. Piotrowski, A. P.; Osuch, M.; Napiorkowski, M. J.; Rowinski, P. M.; Napiorkowski, J. J., Comparing large number of metaheuristics for artificial neural networks training to predict water temperature in a natural river. Computers \& Geosciences 2014, 64, 136-151.

5. Liu, J.; Wu, C.; Wu, G.; Wang, X., A novel differential search algorithm and applications for structure design. Applied Mathematics and Computation 2015, 268, 246-269.

6. Feng, S.; Lu, H.; Tian, P.; Xue, Y.; Lu, J.; Tang, M.; Feng, W., Analysis of microplastics in a remote region of the Tibetan Plateau: Implications for natural environmental response to human activities. Science of The Total Environment 2020, 739, 140087.

7. Fu, X.; Fortino, G.; Pace, P.; Aloi, G.; Li, W., Environment-fusion multipath routing protocol for wireless sensor networks. Information Fusion 2020, 53, 4-19.

8. Han, X.; Zhang, D.; Yan, J.; Zhao, S.; Liu, J., Process development of flue gas desulphurization wastewater treatment in coal-fired power plants towards zero liquid discharge: Energetic, economic and environmental analyses. Journal of Cleaner Production 2020, 261, 121144.

9. He, L.; Chen, Y.; Zhao, H.; Tian, P.; Xue, Y.; Chen, L., Game-based analysis of energy-water nexus for identifying environmental impacts during Shale gas operations under stochastic input. Science of The Total Environment 2018, 627, 1585-1601.

10. He, L.; Shen, J.; Zhang, Y., Ecological vulnerability assessment for ecological conservation and environmental management. Journal of Environmental Management 2018, 206, 1115-1125. 
11. Liu, J.; Liu, Y.; Wang, X., An environmental assessment model of construction and demolition waste based on system dynamics: a case study in Guangzhou. Environmental Science and Pollution Research 2020, 27, (30), 37237-37259.

12. Liu, Y.; Yang, C.; Sun, Q., Thresholds Based Image Extraction Schemes in Big Data Environment in Intelligent Traffic Management. IEEE Transactions on Intelligent Transportation Systems 2020, 1-9.

13. Liu, L.; Li, J.; Yue, F.; Yan, X.; Wang, F.; Bloszies, S.; Wang, Y., Effects of arbuscular mycorrhizal inoculation and biochar amendment on maize growth, cadmium uptake and soil cadmium speciation in Cd-contaminated soil. Chemosphere 2018, 194, 495-503.

14. Yang, Y.; Liu, J.; Yao, J.; Kou, J.; Li, Z.; Wu, T.; Zhang, K.; Zhang, L.; Sun, H., Adsorption behaviors of shale oil in kerogen slit by molecular simulation. Chemical Engineering Journal 2020, 387, 124054.

15. Hu, X.; Chong, H.-Y.; Wang, X., Sustainability perceptions of off-site manufacturing stakeholders in Australia. Journal of Cleaner Production 2019, 227, 346-354.

16. Ghaemi, A.; Rezaie-Balf, M.; Adamowski, J.; Kisi, O.; Quilty, J., On the applicability of maximum overlap discrete wavelet transform integrated with MARS and M5 model tree for monthly pan evaporation prediction. Agricultural and Forest Meteorology 2019, 278, 107647.

17. Keshtegar, B.; Heddam, S.; Sebbar, A.; Zhu, S.-P.; Trung, N.-T., SVR-RSM: a hybrid heuristic method for modeling monthly pan evaporation. Environmental Science and Pollution Research 2019, 26, (35), 35807-35826.

18. Kisi, O.; Heddam, S., Evaporation modelling by heuristic regression approaches using only temperature data. Hydrological Sciences Journal 2019, 64, (6), 653-672.

19. Roy, D. K.; Barzegar, R.; Quilty, J.; Adamowski, J., Using ensembles of adaptive neuro-fuzzy inference system and optimization algorithms to predict reference evapotranspiration in subtropical climatic zones. Journal of Hydrology 2020, 125509.

20. Zhang, B.; Xu, D.; Liu, Y.; Li, F.; Cai, J.; Du, L., Multi-scale evapotranspiration of summer maize and the controlling meteorological factors in north China. Agricultural and Forest Meteorology 2016, 216, 1-12.

21. Chao, L.; Zhang, K.; Li, Z.; Zhu, Y.; Wang, J.; Yu, Z., Geographically weighted regression based methods for merging satellite and gauge precipitation. Journal of Hydrology 2018, 558, 275-289.

22. Chen, Y.; He, L.; Guan, Y.; Lu, H.; Li, J., Life cycle assessment of greenhouse gas emissions and waterenergy optimization for shale gas supply chain planning based on multi-level approach: Case study in Barnett, Marcellus, Fayetteville, and Haynesville shales. Energy Conversion and Management 2017, 134, 382-398.

23. He, L.; Chen, Y.; Li, J., A three-level framework for balancing the tradeoffs among the energy, water, and air-emission implications within the life-cycle shale gas supply chains. Resources, Conservation and Recycling 2018, 133, 206-228.

24. Lu, H.; Tian, P.; He, L., Evaluating the global potential of aquifer thermal energy storage and determining the potential worldwide hotspots driven by socio-economic, geo-hydrologic and climatic conditions. Renewable and Sustainable Energy Reviews 2019, 112, 788-796.

25. Wang, Y.; Yao, M.; Ma, R.; Yuan, Q.; Yang, D.; Cui, B.; Ma, C.; Liu, M.; Hu, D., Design strategy of barium titanate/polyvinylidene fluoride-based nanocomposite films for high energy storage. Journal of Materials Chemistry A 2020, 8, (3), 884-917.

26. Zhang, W., Parameter Adjustment Strategy and Experimental Development of Hydraulic System for Wave Energy Power Generation. Symmetry 2020, 12, (5), 711. 
27. Zhao, X.; Ye, Y.; Ma, J.; Shi, P.; Chen, H., Construction of electric vehicle driving cycle for studying electric vehicle energy consumption and equivalent emissions. Environmental Science and Pollution Research 2020, 1-15.

28. Zhu, L.; Kong, L.; Zhang, C., Numerical Study on Hysteretic Behaviour of Horizontal-Connection and Energy-Dissipation Structures Developed for Prefabricated Shear Walls. Applied Sciences 2020, 10, (4), 1240 .

29. Deng, Y.; Zhang, T.; Sharma, B. K.; Nie, H., Optimization and mechanism studies on cell disruption and phosphorus recovery from microalgae with magnesium modified hydrochar in assisted hydrothermal system. Science of The Total Environment 2019, 646, 1140-1154.

30. Zhang, T.; Wu, X.; Fan, X.; Tsang, D. C. W.; Li, G.; Shen, Y., Corn waste valorization to generate activated hydrochar to recover ammonium nitrogen from compost leachate by hydrothermal assisted pretreatment. Journal of Environmental Management 2019, 236, 108-117.

31. Li, Z.-G.; Cheng, H.; Gu, T.-Y., Research on dynamic relationship between natural gas consumption and economic growth in China. Structural Change and Economic Dynamics 2019, 49, 334-339.

32. Liu, E.; Lv, L.; Yi, Y.; Xie, P., Research on the Steady Operation Optimization Model of Natural Gas Pipeline Considering the Combined Operation of Air Coolers and Compressors. IEEE Access 2019, 7, 83251-83265.

33. Su, Z.; Liu, E.; Xu, Y.; Xie, P.; Shang, C.; Zhu, Q., Flow field and noise characteristics of manifold in natural gas transportation station. Oil E Gas Science and Technology-Revue d'IFP Energies nouvelles 2019, 74,70 .

34. Chen, Y.; Li, J.; Lu, H.; Yan, P., Coupling system dynamics analysis and risk aversion programming for optimizing the mixed noise-driven shale gas-water supply chains. Journal of Cleaner Production 2021, 278, 123209.

35. Cheng, X.; He, L.; Lu, H.; Chen, Y.; Ren, L., Optimal water resources management and system benefit for the Marcellus shale-gas reservoir in Pennsylvania and West Virginia. Journal of Hydrology 2016, 540, 412-422.

36. Li, L.-L.; Liu, Y.-W.; Tseng, M.-L.; Lin, G.-Q.; Ali, M. H., Reducing environmental pollution and fuel consumption using optimization algorithm to develop combined cooling heating and power system operation strategies. Journal of Cleaner Production 2020, 247, 119082.

37. Li, X.; Zhang, R.; Zhang, X.; Zhu, P.; Yao, T., Silver-Catalyzed Decarboxylative Allylation of Difluoroarylacetic Acids with Allyl Sulfones in Water. Chemistry - An Asian Journal 2020, 15, (7), 1175 1179.

38. Quan, Q.; Hao, Z.; Xifeng, H.; Jingchun, L., Research on water temperature prediction based on improved support vector regression. Neural Computing and Applications 2020, 1-10.

39. Yang, M.; Sowmya, A., An Underwater Color Image Quality Evaluation Metric. IEEE Transactions on Image Processing 2015, 24, (12), 6062-6071.

40. Cao, B.; Fan, S.; Zhao, J.; Yang, P.; Muhammad, K.; Tanveer, M., Quantum-enhanced multiobjective large-scale optimization via parallelism. Swarm and Evolutionary Computation 2020, 57, 100697.

41. Jia, L.; Liu, B.; Zhao, Y.; Chen, W.; Mou, D.; Fu, J.; Wang, Y.; Xin, W.; Zhao, L., Structure design of MoS2@Mo2C on nitrogen-doped carbon for enhanced alkaline hydrogen evolution reaction. Journal of Materials Science 2020, 55, (34), 16197-16210.

42. Lyu, Z.; Chai, J.; Xu, Z.; Qin, Y.; Cao, J., A Comprehensive Review on Reasons for Tailings Dam Failures Based on Case History. Advances in Civil Engineering 2019, 2019, 4159306. 
43. Feng, W.; Lu, H.; Yao, T.; Yu, Q., Drought characteristics and its elevation dependence in the QinghaiTibet plateau during the last half-century. Scientific Reports 2020, 10, (1), 14323.

44. Qian, J.; Feng, S.; Li, Y.; Tao, T.; Han, J.; Chen, Q.; Zuo, C., Single-shot absolute 3D shape measurement with deep-learning-based color fringe projection profilometry. Optics Letters 2020, 45, (7), 1842-1845.

45. Zenggang, X.; Zhiwen, T.; Xiaowen, C.; Xue-min, Z.; Kaibin, Z.; Conghuan, Y., Research on Image Retrieval Algorithm Based on Combination of Color and Shape Features. Journal of Signal Processing Systems 2019, 1-8.

46. Zhu, Q., Research on Road Traffic Situation Awareness System Based on Image Big Data. IEEE Intelligent Systems 2020, 35, (1), 18-26.

47. Xu, S.; Wang, J.; Shou, W.; Ngo, T.; Sadick, A.-M.; Wang, X., Computer Vision Techniques in Construction: A Critical Review. Archives of Computational Methods in Engineering 2020.

48. Yan, J.; Pu, W.; Zhou, S.; Liu, H.; Bao, Z., Collaborative detection and power allocation framework for target tracking in multiple radar system. Information Fusion 2020, 55, 173-183.

49. Chao, M.; Kai, C.; Zhiwei, Z., Research on tobacco foreign body detection device based on machine vision. Transactions of the Institute of Measurement and Control 2020, 42, (15), 2857-2871.

50. Liu, D.; Wang, S.; Huang, D.; Deng, G.; Zeng, F.; Chen, H., Medical image classification using spatial adjacent histogram based on adaptive local binary patterns. Computers in biology and medicine 2016, 72, 185-200.

51. Zhang, X.; Jiang, R.; Wang, T.; Wang, J., Recursive Neural Network for Video Deblurring. IEEE Transactions on Circuits and Systems for Video Technology 2020, 1-1.

52. Yu, H.; Shen, S.; Qian, G.; Gong, X., Packing theory and volumetrics-based aggregate gradation design method. Journal of Materials in Civil Engineering 2020, 32, (6), 04020110.

53. Zhang, C.; Wang, H., Swing vibration control of suspended structures using the Active Rotary Inertia Driver system: Theoretical modeling and experimental verification. Structural Control and Health Monitoring 2020, 27, (6), e2543.

54. Sun, Y.; Wang, J.; Wu, J.; Shi, W.; Ji, D.; Wang, X.; Zhao, X., Constraints hindering the development of high-rise modular buildings. Applied Sciences 2020, 10, (20), 7159.

55. Abedini, M.; Mutalib, A. A.; Zhang, C.; Mehrmashhadi, J.; Raman, S. N.; Alipour, R.; Momeni, T.; Mussa, M. H., Large deflection behavior effect in reinforced concrete columns exposed to extreme dynamic loads. Frontiers of Structural and Civil Engineering 2020, 14, (2), 532-553.

56. Mou, B.; Li, X.; Bai, Y.; Wang, L., Shear behavior of panel zones in steel beam-to-column connections with unequal depth of outer annular stiffener. Journal of Structural Engineering 2019, 145, (2), 04018247.

57. Zhang, K.; Ruben, G. B.; Li, X.; Li, Z.; Yu, Z.; Xia, J.; Dong, Z., A comprehensive assessment framework for quantifying climatic and anthropogenic contributions to streamflow changes: A case study in a typical semi-arid North China basin. Environmental Modelling $\mathcal{E}$ Software 2020, 128, 104704.

58. Qian, J.; Feng, S.; Tao, T.; Hu, Y.; Li, Y.; Chen, Q.; Zuo, C., Deep-learning-enabled geometric constraints and phase unwrapping for single-shot absolute 3D shape measurement. APL Photonics 2020, 5, (4), 046105.

59. Yang, W.; Pudasainee, D.; Gupta, R.; Li, W.; Wang, B.; Sun, L., An overview of inorganic particulate matter emission from coal/biomass/MSW combustion: Sampling and measurement, formation, distribution, inorganic composition and influencing factors. Fuel Processing Technology 2020, 106657.

60. Zhang, C.-W.; Ou, J.-P.; Zhang, J.-Q., Parameter optimization and analysis of a vehicle suspension system controlled by magnetorheological fluid dampers. Structural Control and Health Monitoring 2006, $13,(5), 885-896$ 
61. Mou, B.; Zhao, F.; Qiao, Q.; Wang, L.; Li, H.; He, B.; Hao, Z., Flexural behavior of beam to column joints with or without an overlying concrete slab. Engineering Structures 2019, 199, 109616.

62. Abedini, M.; Zhang, C., Performance Assessment of Concrete and Steel Material Models in LS-DYNA for Enhanced Numerical Simulation, A State of the Art Review. Archives of Computational Methods in Engineering 2020.

63. Gholipour, G.; Zhang, C.; Mousavi, A. A., Numerical analysis of axially loaded RC columns subjected to the combination of impact and blast loads. Engineering Structures 2020, 219, 110924.

64. Zhang, C.; Abedini, M.; Mehrmashhadi, J., Development of pressure-impulse models and residual capacity assessment of RC columns using high fidelity Arbitrary Lagrangian-Eulerian simulation. Engineering Structures 2020, 224, 111219.

65. Yue, H.; Wang, H.; Chen, H.; Cai, K.; Jin, Y., Automatic detection of feather defects using Lie group and fuzzy Fisher criterion for shuttlecock production. Mechanical Systems and Signal Processing 2020, 141, 106690.

66. Zhu, G.; Wang, S.; Sun, L.; Ge, W.; Zhang, X., Output Feedback Adaptive Dynamic Surface SlidingMode Control for Quadrotor UAVs with Tracking Error Constraints. Complexity 2020, 2020, 8537198.

67. Xiong, Q.; Zhang, X.; Wang, W.-F.; Gu, Y., A Parallel Algorithm Framework for Feature Extraction of EEG Signals on MPI. Computational and Mathematical Methods in Medicine 2020, 2020, 9812019.

68. Zhang, H.; Qu, S.; Li, H.; Luo, J.; Xu, W., A Moving Shadow Elimination Method Based on Fusion of Multi-Feature. IEEE Access 2020, 8, 63971-63982.

69. Zhang, J.; Liu, B., A review on the recent developments of sequence-based protein feature extraction methods. Current Bioinformatics 2019, 14, (3), 190-199.

70. Zhang, X.; Fan, M.; Wang, D.; Zhou, P.; Tao, D., Top-k Feature Selection Framework Using Robust 0-1 Integer Programming. IEEE Transactions on Neural Networks and Learning Systems 2020, 1-15.

71. Zhao, X.; Li, D.; Yang, B.; Chen, H.; Yang, X.; Yu, C.; Liu, S., A two-stage feature selection method with its application. Computers $\mathcal{E}$ Electrical Engineering 2015, 47, 114-125.

72. Liu, S.; Chan, F. T. S.; Ran, W., Decision making for the selection of cloud vendor: An improved approach under group decision-making with integrated weights and objective/subjective attributes. Expert Systems with Applications 2016, 55, 37-47.

73. Tian, P.; Lu, H.; Feng, W.; Guan, Y.; Xue, Y., Large decrease in streamflow and sediment load of Qinghai-Tibetan Plateau driven by future climate change: A case study in Lhasa River Basin. CATENA 2020, 187, 104340.

74. Yang, L.; Chen, H., Fault diagnosis of gearbox based on RBF-PF and particle swarm optimization wavelet neural network. Neural Computing and Applications 2019, 31, (9), 4463-4478.

75. Cao, B.; Zhao, J.; Lv, Z.; Gu, Y.; Yang, P.; Halgamuge, S. K., Multiobjective Evolution of Fuzzy Rough Neural Network via Distributed Parallelism for Stock Prediction. IEEE Transactions on Fuzzy Systems 2020, 28, (5), 939-952.

76. Shi, K.; Wang, J.; Tang, Y.; Zhong, S., Reliable asynchronous sampled-data filtering of T-S fuzzy uncertain delayed neural networks with stochastic switched topologies. Fuzzy Sets and Systems 2020, 381, 1-25.

77. Shi, K.; Wang, J.; Zhong, S.; Tang, Y.; Cheng, J., Non-fragile memory filtering of T-S fuzzy delayed neural networks based on switched fuzzy sampled-data control. Fuzzy Sets and Systems 2020, 394, 4064.

78. Yang, S.; Deng, B.; Wang, J.; Li, H.; Lu, M.; Che, Y.; Wei, X.; Loparo, K. A., Scalable Digital Neuromorphic Architecture for Large-Scale Biophysically Meaningful Neural Network With Multi- 
Compartment Neurons. IEEE Transactions on Neural Networks and Learning Systems 2020, 31, (1), 148162.

79. Adeli, H., Neural networks in civil engineering: 1989-2000. Computer-Aided Civil and Infrastructure Engineering 2001, 16, (2), 126-142.

80. Hornik, K.; Stinchcombe, M.; White, H., Multilayer feedforward networks are universal approximators. Neural networks 1989, 2, (5), 359-366.

81. Lv, Z.; Qiao, L., Deep belief network and linear perceptron based cognitive computing for collaborative robots. Applied Soft Computing 2020, 92, 106300.

82. Xu, M.; Li, T.; Wang, Z.; Deng, X.; Yang, R.; Guan, Z., Reducing Complexity of HEVC: A Deep Learning Approach. IEEE Transactions on Image Processing 2018, 27, (10), 5044-5059.

83. Li, T.; Xu, M.; Zhu, C.; Yang, R.; Wang, Z.; Guan, Z., A Deep Learning Approach for Multi-Frame InLoop Filter of HEVC. IEEE Transactions on Image Processing 2019, 28, (11), 5663-5678.

84. Qiu, T.; Shi, X.; Wang, J.; Li, Y.; Qu, S.; Cheng, Q.; Cui, T.; Sui, S., Deep Learning: A Rapid and Efficient Route to Automatic Metasurface Design. Advanced Science 2019, 6, (12), 1900128.

85. Chen, H.; Chen, A.; Xu, L.; Xie, H.; Qiao, H.; Lin, Q.; Cai, K., A deep learning CNN architecture applied in smart near-infrared analysis of water pollution for agricultural irrigation resources. Agricultural Water Management 2020, 240, 106303.

86. Chen, H.-L.; Wang, G.; Ma, C.; Cai, Z.-N.; Liu, W.-B.; Wang, S.-J., An efficient hybrid kernel extreme learning machine approach for early diagnosis of Parkinson's disease. Neurocomputing 2016, 184, 131144.

87. Hu, L.; Hong, G.; Ma, J.; Wang, X.; Chen, H., An efficient machine learning approach for diagnosis of paraquat-poisoned patients. Computers in Biology and Medicine 2015, 59, 116-124.

88. Wang, S.-J.; Chen, H.-L.; Yan, W.-J.; Chen, Y.-H.; Fu, X., Face recognition and micro-expression recognition based on discriminant tensor subspace analysis plus extreme learning machine. Neural processing letters 2014, 39, (1), 25-43.

89. Xia, J.; Chen, H.; Li, Q.; Zhou, M.; Chen, L.; Cai, Z.; Fang, Y.; Zhou, H., Ultrasound-based differentiation of malignant and benign thyroid Nodules: An extreme learning machine approach. Computer methods and programs in biomedicine 2017, 147, 37-49.

90. Wojtkiewicz, J.; Katragadda, S.; Gottumukkala, R. In A Concept-Drift Based Predictive-Analytics Framework: Application for Real-Time Solar Irradiance Forecasting, 2018 IEEE International Conference on Big Data (Big Data), 2018; IEEE: pp 5462-5464.

91. Lee, J.; Wang, W.; Harrou, F.; Sun, Y., Reliable solar irradiance prediction using ensemble learningbased models: A comparative study. Energy Conversion and Management 2020, 208, 112582.

92. Wang, H.; Sun, J.; Wang, W., Photovoltaic Power Forecasting Based on EEMD and a Variable-Weight Combination Forecasting Model. Sustainability 2018, 10, (8), 2627.

93. Massimo, A.; Dell'Isola, M.; Frattolillo, A.; Ficco, G., Development of a geographical information system (GIS) for the integration of solar energy in the energy planning of a wide area. Sustainability 2014, 6, (9), 5730-5744.

94. Salcedo-Sanz, S.; Cornejo-Bueno, L.; Prieto, L.; Paredes, D.; García-Herrera, R., Feature selection in machine learning prediction systems for renewable energy applications. Renewable and Sustainable Energy Reviews 2018, 90, 728-741.

95. Barrera, J. M.; Reina, A.; Maté, A.; Trujillo, J. C., Solar Energy Prediction Model Based on Artificial Neural Networks and Open Data. Sustainability 2020, 12, (17), 6915. 
96. Yaïci, W.; Longo, M.; Entchev, E.; Foiadelli, F., Simulation study on the effect of reduced inputs of artificial neural networks on the predictive performance of the solar energy system. Sustainability 2017, 9, (8), 1382.

97. Yadav, A. K.; Malik, H.; Chandel, S., Application of rapid miner in ANN based prediction of solar radiation for assessment of solar energy resource potential of 76 sites in Northwestern India. Renewable and Sustainable Energy Reviews 2015, 52, 1093-1106.

98. Meenal, R.; Selvakumar, A. I., Assessment of SVM, empirical and ANN based solar radiation prediction models with most influencing input parameters. Renewable Energy 2018, 121, 324-343.

99. Mohammadi, K.; Shamshirband, S.; Kamsin, A.; Lai, P.; Mansor, Z., Identifying the most significant input parameters for predicting global solar radiation using an ANFIS selection procedure. Renewable and Sustainable Energy Reviews 2016, 63, 423-434.

100. Quej, V. H.; Almorox, J.; Arnaldo, J. A.; Saito, L., ANFIS, SVM and ANN soft-computing techniques to estimate daily global solar radiation in a warm sub-humid environment. Journal of Atmospheric and Solar-Terrestrial Physics 2017, 155, 62-70.

101. Houssein, E. H., Machine Learning and Meta-heuristic Algorithms for Renewable Energy: A Systematic Review. In Advanced Control and Optimization Paradigms for Wind Energy Systems, Springer: 2019; pp 165187.

102. Liu, H.; Chen, C.; Lv, X.; Wu, X.; Liu, M., Deterministic wind energy forecasting: A review of intelligent predictors and auxiliary methods. Energy Conversion and Management 2019, 195, 328-345.

103. Elsheikh, A. H.; Sharshir, S. W.; Abd Elaziz, M.; Kabeel, A.; Guilan, W.; Haiou, Z., Modeling of solar energy systems using artificial neural network: A comprehensive review. Solar Energy 2019, 180, 622639.

104. Akhter, M. N.; Mekhilef, S.; Mokhlis, H.; Shah, N. M., Review on forecasting of photovoltaic power generation based on machine learning and metaheuristic techniques. IET Renewable Power Generation 2019, 13, (7), 1009-1023.

105. Moayedi, H.; Mehrabi, M.; Mosallanezhad, M.; Rashid, A. S. A.; Pradhan, B., Modification of landslide susceptibility mapping using optimized PSO-ANN technique. Engineering with Computers 2019, 35, (3), 967-984.

106. Abedinia, O.; Amjady, N.; Ghadimi, N., Solar energy forecasting based on hybrid neural network and improved metaheuristic algorithm. Computational Intelligence 2018, 34, (1), 241-260.

107. Galván, I. M.; Valls, J. M.; Cervantes, A.; Aler, R., Multi-objective evolutionary optimization of prediction intervals for solar energy forecasting with neural networks. Information Sciences 2017, 418, 363-382.

108. Halabi, L. M.; Mekhilef, S.; Hossain, M., Performance evaluation of hybrid adaptive neuro-fuzzy inference system models for predicting monthly global solar radiation. Applied Energy 2018, 213, 247261.

109. Vaisakh, T.; Jayabarathi, R., Analysis on intelligent machine learning enabled with meta-heuristic algorithms for solar irradiance prediction. Evolutionary Intelligence 2020, 1-20.

110. Louzazni, M.; Khouya, A.; Amechnoue, K.; Gandelli, A.; Mussetta, M.; Crăciunescu, A., Metaheuristic algorithm for photovoltaic parameters: comparative study and prediction with a firefly algorithm. Applied Sciences 2018, 8, (3), 339.

111. Bechouat, M.; Younsi, A.; Sedraoui, M.; Soufi, Y.; Yousfi, L.; Tabet, I.; Touafek, K., Parameters identification of a photovoltaic module in a thermal system using meta-heuristic optimization methods. International Journal of Energy and Environmental Engineering 2017, 8, (4), 331-341. 
112. Abdalla, O.; Rezk, H.; Ahmed, E. M., Wind driven optimization algorithm based global MPPT for PV system under non-uniform solar irradiance. Solar Energy 2019, 180, 429-444.

113. Elsheikh, A.; Abd Elaziz, M., Review on applications of particle swarm optimization in solar energy systems. International Journal of Environmental Science and Technology 2019, 16, (2), 1159-1170.

114. Aybar-Ruiz, A.; Jiménez-Fernández, S.; Cornejo-Bueno, L.; Casanova-Mateo, C.; Sanz-Justo, J.; Salvador-González, P.; Salcedo-Sanz, S., A novel grouping genetic algorithm-extreme learning machine approach for global solar radiation prediction from numerical weather models inputs. Solar Energy 2016, 132, 129-142.

115. Khosravi, A.; Syri, S., Modeling of geothermal power system equipped with absorption refrigeration and solar energy using multilayer perceptron neural network optimized with imperialist competitive algorithm. Journal of Cleaner Production 2020, 276, 124216.

116. Song, Y.; Lu, J.; Lu, H.; Zhang, G., Fuzzy clustering-based adaptive regression for drifting data streams. IEEE Transactions on Fuzzy Systems 2019, 28, (3), 544-557.

117. Kamble, M.; Ghosh, S.; Patel, P. In Solar Irradiance Prediction using meteorological data by ensemble models, 2nd International Conference on Data, Engineering and Applications (IDEA), 2020; IEEE: pp 1-6.

118. Abedinpourshotorban, H.; Shamsuddin, S. M.; Beheshti, Z.; Jawawi, D. N., Electromagnetic field optimization: A physics-inspired metaheuristic optimization algorithm. Swarm and Evolutionary Computation 2016, 26, 8-22.

119. Bouchekara, H.; Zellagui, M.; Abido, M. A., Optimal coordination of directional overcurrent relays using a modified electromagnetic field optimization algorithm. Applied Soft Computing 2017, 54, 267 283.

120. Bouchekara, H., Solution of the optimal power flow problem considering security constraints using an improved chaotic electromagnetic field optimization algorithm. Neural Computing and Applications 2020, $32,(7), 2683-2703$.

121. Duan, Q.; Gupta, V. K.; Sorooshian, S., Shuffled complex evolution approach for effective and efficient global minimization. Journal of optimization theory and applications 1993, 76, (3), 501-521.

122. Eusuff, M. M.; Lansey, K. E., Optimization of water distribution network design using the shuffled frog leaping algorithm. Journal of Water Resources planning and management 2003, 129, (3), 210-225.

123. Zheng, S.; Lyu, Z.; Foong, L. K., Early prediction of cooling load in energy-efficient buildings through novel optimizer of shuffled complex evolution. Engineering with Computers 2020, 1-15.

124. Ma, X.; Foong, L. K.; Morasaei, A.; Ghabussi, A.; Lyu, Z., Swarm-based hybridizations of neural network for predicting the concrete strength. Smart Structures and Systems 2020, 26, (2), 241-251.

125. Gao, X.; Cui, Y.; Hu, J.; Xu, G.; Wang, Z.; Qu, J.; Wang, H., Parameter extraction of solar cell models using improved shuffled complex evolution algorithm. Energy conversion and management 2018, 157, 460-479.

126. Moayedi, H.; Bui, D. T.; Thi Ngo, P. T., Shuffled frog leaping algorithm and wind-driven optimization technique modified with multilayer perceptron. Applied Sciences 2020, 10, (2), 689.

127. Majeed, K.; Qyyum, M. A.; Nawaz, A.; Ahmad, A.; Naqvi, M.; He, T.; Lee, M., Shuffled Complex Evolution-Based Performance Enhancement and Analysis of Cascade Liquefaction Process for LargeScale LNG Production. Energies 2020, 13, (10), 2511.

128. Bayat, P.; Afrakhte, H., A purpose-oriented shuffled complex evolution optimization algorithm for energy management of multi-microgrid systems considering outage duration uncertainty. Journal of Intelligent E Fuzzy Systems 2020, (Preprint), 1-18. 
129. Siahbalaee, J.; Rezanejad, N.; Gharehpetian, G. B., Reconfiguration and DG Sizing and Placement Using Improved Shuffled Frog Leaping Algorithm. Electric Power Components and Systems 2020, 1-14.

130. Gandhi, B. R.; Bhattacharjya, R., Introduction to Shuffled Frog Leaping Algorithm and Its Sensitivity to the Parameters of the Algorithm. In Nature-Inspired Methods for Metaheuristics Optimization, Springer: 2020; pp 105-117. 